\title{
Desarrollo de un sistema de comunicación remota vía modem con un pc que se encuentra apagado ${ }^{1}$ Remote communication system development via modem with a switched off desktop
}

Fecha de recepción: 28 de marzo de 2008

Fecha de aceptación: 14 de julio de 2008

Darin Jairo Mosquera Palacios ${ }^{2}$, Octavio Salcedo Parra ${ }^{3}$, Marlon Patiño Bernal $^{4}$

\section{Resumen}

El presente artículo, describe el diseño y desarrollo de una tarjeta para el encendido de un PC remoto vía MODEM como resultado parcial del proyecto de investigación Sistema de gestión en línea de un PC por Internet, donde se comunican dos computadores por Internet y se suple uno de los obstáculos que se presenta cuando una de las máquinas se encuentra físicamente distante y apagada. Lo que busca este trabajo es no preocuparse por la máquina remota a gestionar, si no por qué tipo de información es la que se necesita intercambiar o software a instalar.

\section{Palabras clave}

Detector de llamada, emulador de descuelgue, identificador de decodificador, sistema de encendido, opto acoplador.

\section{ABSTRACT}

This article is a partial result of the research project "Sistema de Gestion en Línea de un PC por Internet" where two computers communicate themselves on Internet, and overcomes one of the obstacles that comes up when one of the equipments is physically distant and turned off. Willing to facilitate information interchange, this work aims to wipe out this hindrance by avoiding worming about the remote equipment, but about the kind or information and/or the software set up required.

\section{Key words}

Detector of call, emulator of offs-hook, identifier of decoder, ignition system.

1 Remote communication via modem with a pc that is dull

2 Ingeniero de Sistemas, Especialista en Teleinformática, Magíster en Teleinformática de la Universidad Distrital Francisco José de Caldas, Profesor adscrito a la facultad tecnológica de esta Universidad, Director del grupo de Investigación en el área de Telemática "Orión" de la misma universidad. djmosquerap@udistrital.edu.co

3 Ingeniero de Sistemas, Magíster en Teleinformática de la Universidad Distrital Francisco José de Caldas. ojsalcedop@unal.edu.co

4 Ingeniero Electrónico, Especialista en Telecomunicaciones Móviles, Universidad Distrital Francisco José de Caldas (F.J.C),, Magíster en ingenieria de Telecomunicaciones, Universidad Nacional de Colombia, Docente Universidad Distrital F.J.C. adscrito a la Facultad Tecnológica. marlonpb@udistrital.edu.co 


\section{Introducción}

Las redes, en general, consisten en compartir recursos; uno de sus beneficios es hacer que todos los programas, datos y equipos estén disponibles para cualquiera en la red, que así lo solicite, sin importar la localización física del recurso [1][2], teniendo en cuenta que el computador [3] al cual se quiere comunicar se encuentre encendido. Por ello, se da a conocer cómo podemos comunicarnos con un PC que se encuentra apagado, mediante la adición de una tarjeta externa que se conecta a la terminal telefónica y al MODEM del equipo, para facilitar su normal funcionamiento de acuerdo con sus estándares[4].

El artículo está compuesto por tres secciones: Antecedentes, en donde se plasma una de las dificultades que se presenta cuando se desea comunicar con un PC vía MODEM que no se vea en la red y/o está apagado; Diseño y principio de funcionamiento, en donde se describe el desarrollo y funcionamiento de la tarjeta y por último, Requisitos de interfaces, en donde explica la forma de operabilidad de la tarjeta.

\section{Antecedentes}

Desde las primeras computadoras [5], el hombre ha visto la necesidad de comunicarlas e intercambiar información dentro de una misma red o medio que lo permita [6]; la dificultad consistía en la inexistencia de una red global; con la red de redes Internet[7], se pueden conectar dos o más computadores por diferentes vías, esto es posible por la familia de protocolos de TCP/IP y la red digital de servicios integrado RDSI[8], donde se reciben los datos por un modem que, conectado a la línea telefónica, los procesa y los reenvía; este proceso se realiza mientras el' PC se encuentre activo. Algunas motherboard, como la ATX o la $\mathrm{BIOS}^{5}$, presentan una opción de encendido remoto, lo que permite que por una señal el ordenador se active aunque las dos máquinas no necesariamente tengan el mismo sistema operativo. Con el diseño de la tarjeta, la motherboard y el sistema operativo son transparentes al usuario.

\section{Principio de funcionamiento}

Debido a los avances tecnológicos, una comunicación vía MODEM se puede hacer en cualquier momento y en todo lugar sin importar si el PC remoto, al cual se quiere comunicar, esté o no encendido[9].

En este artículo se logra comunicar dos computadores remotamente, mediante señales transmitidas a través de la RTF (Red Telefónica Conmutada) en forma serial. Estos datos iniciales van a estar almacenados en la tarjeta temporalmente y validados por el software programado en lenguaje ensamblador (Asembler)[10][11] para su control y autenticación regida bajo estándares internacionales.

La tarjeta cuenta con una identificación única: la dirección física está formada por 32 bits y, al igual que las tarjetas de red, se representa por medio de 6 bits hexadecimales de tipo 00-00-0D-1A-12-35, de los cuales los 3 primeros ( 24 bits), denominados Identificador Organizacional Único (UOI) [12] son asignados al fabricante concreto y los 3 últimos ( 24 bits) son asignados secuencialmente. El software, desarrollado e instalado al lado del cliente, controla

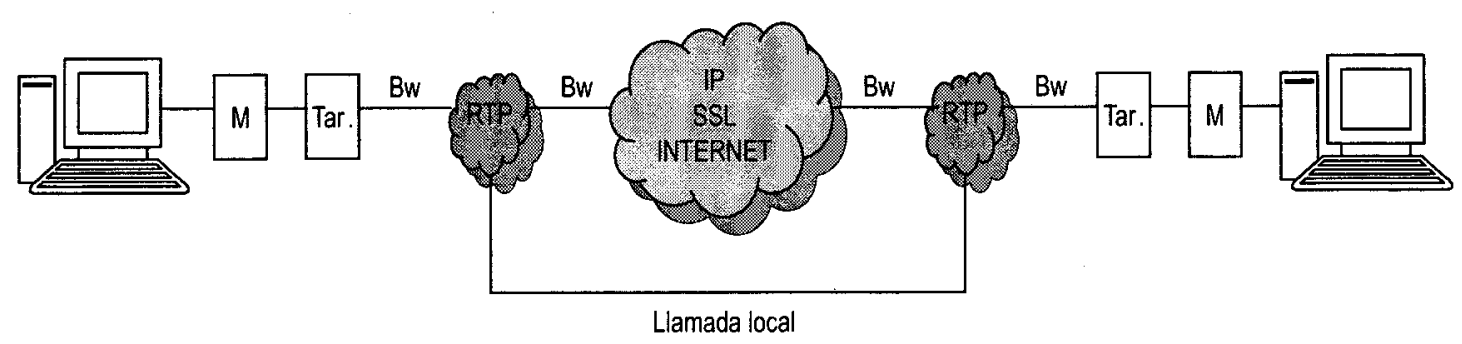

Figura 1. Diagrama general sistema de gestión en linea de un $P C$ por Internet

5 Basic Input/output System 


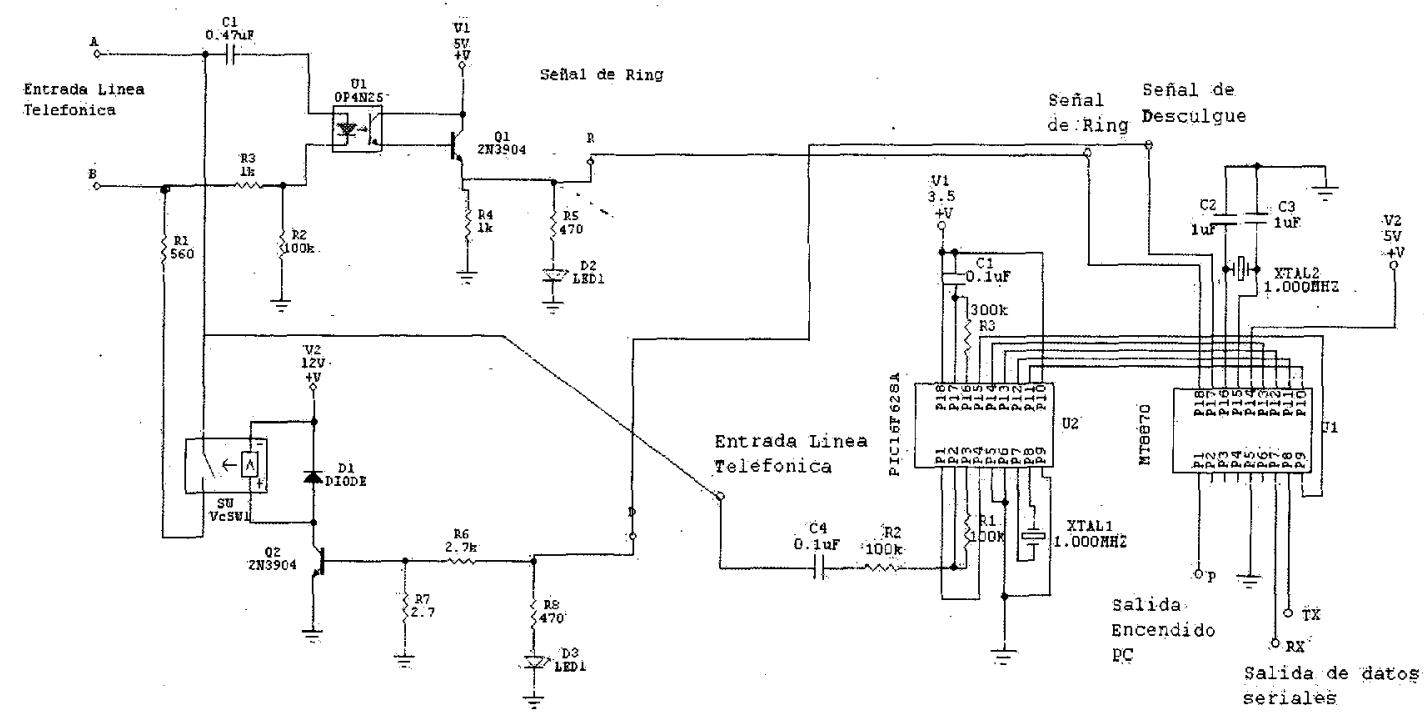

Figura 2. Diseño de la tarjeta

el acceso al PC y almacena temporalmente los datos que son recibidos en forma serial [12] (dirección ip del equipo gestor, clave) y los envía al equipo remoto por intermedio del MODEM(ver cuadro 5).

La figura 1 describe un diagrama general del proyecto (sistema de gestión en línea de un PC por Internet) en donde aparecen dos computadores ubicados en sitios remotos con sus respectivos MODEM (M) y conectados a ellos la(s) tarjeta(s) (tar.) que, dependiendo de la cantidad de tonos, determina si es para encender el PC o si es una llamada normal, y conectados a la Red Telefónica Pública (RTP) e Internet (IP,SSL ${ }^{6}$ ), establece la comunicación[13] y empieza a gestionar el equipo.

Cuando la línea telefónica se encuentra en reposo (teléfono colgado) mide en sus terminales entre $45 \mathrm{v}-55 \mathrm{v}$. El circuito muestra la forma como se detecta la señal de ring de una línea telefónica, para ello se utilizan dos resistencias (R3 y R2) que atenúan el voltaje de la línea para ser aplicada a un Opto acoplador que es el encargado de proteger el circuito compuesto por la línea telefónica y el sistema desarrollado. A la salida del opto acoplador encontramos un transistor configurado en corte y saturación que logra una señal digital de $0 \mathrm{~V}$ y $5 \mathrm{~V}$, esta señal es enviada a el microprocesador para ser

6 IP Protocolo Ithernet SSL Secure Sockets Layer identificada; si la identificación es correcta (5 ring) el microcontrolador entrega un pulso digital por el PIN RA0 (PIN 17), que activa un transistor configurado en corte y saturación para cerrar el contacto (RELEVO). Al cerrar un circuito compuesto por una resistencia de 560 Ohmios, entre los hilos de la línea telefónica (terminales RJ11), se emula el descuelgue de la misma.

Una vez el proceso de llamada haya sido concretado, el usuario remoto debe digital la clave a través del aparato telefónico, para ello la tarjeta consta de un circuito integrado (MT8870) que decodifica los tonos de la línea telefónica y los convierte a cuatro dígitos binarios; este IC puede detectar cuando el usuario presiona un nuevo dígito en el aparato telefónico (PIN 15). Los datos binarios son entregados al microcontrolador y este es el encargado de confirmar si la clave digitada es correcta. Si es así, el microcontrolador envía una señal digital de confirmación la cual debe encender el equipo de cómputo (para esto se puede utilizar un transistor en corte y saturación, un relevo o simplemente un sistema de detección) y queda en espera de recibir la dirección IP del equipo remoto para ser transmitida, esta dirección IP debe ser digital; como cada octeto esta separado por un punto, este deberá ser remplazado por el símbolo asterisco $\left(^{*}\right)$. Cuando termine de transmitir los dígitos se deberá terminar la llamada con la tecla numeral (\#). En este 


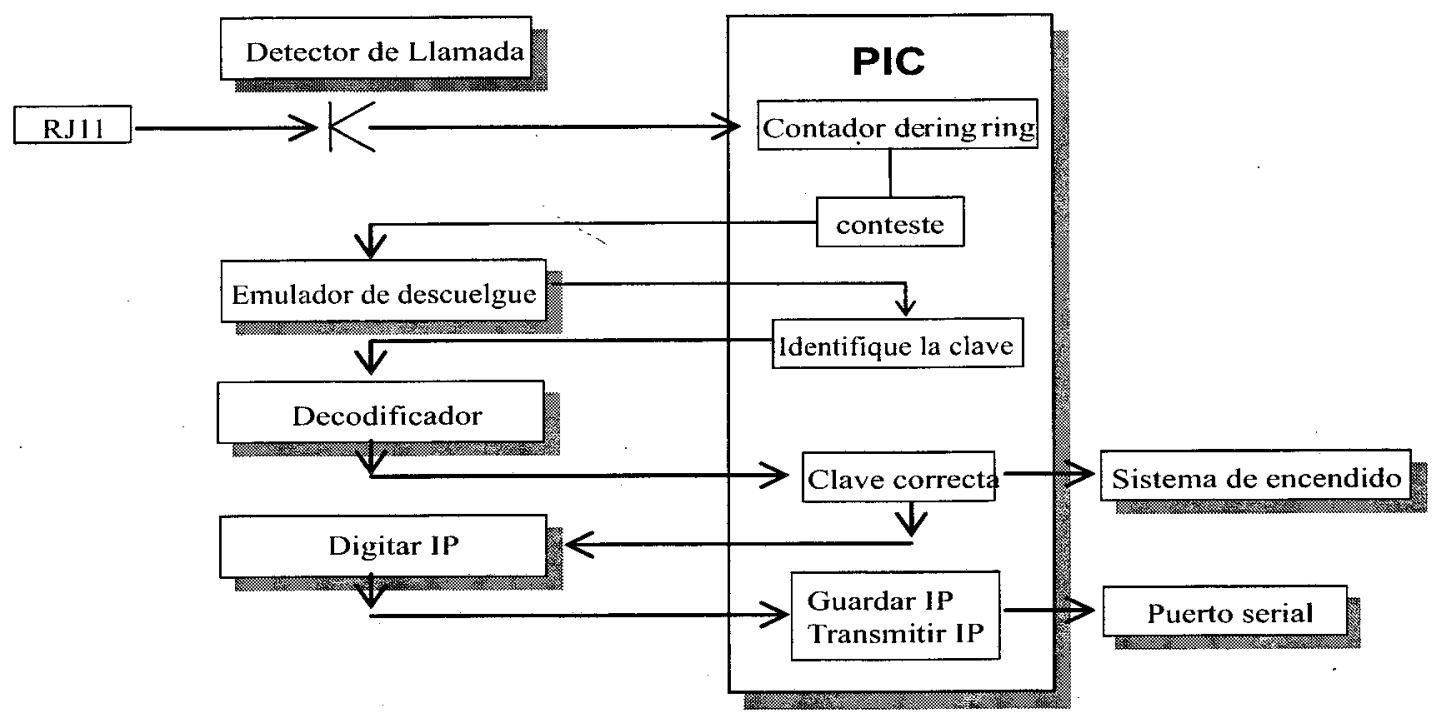

Figura 3. Diagrama funcional de la tarjeta

momento el microcontrolador empezará a trasmitir la dirección IP hacia el equipo de cómputo local, de forma asíncrona, a través del puerto serial utilizando el estándar Rs232.

La figura 3 hace una descripción del funcionamiento de la tarjeta de encendido de un PC

Esta tarjeta tiene cuatro subsistemas importantes:

\section{Detector de llamada}

La tarjeta debe estar conectada a la línea telefónica por medio de un conector RJ11 (ver figura 2) que trabajará a $5 \mathrm{v}$. El detector de llamada es un sistema que consta de un switch, resistencias y un led, de manera que cuando se efectúa la llamada desde el computador gestor (un punto geográficamente distante) el sistema detector adaptará la señal de timbre que provee el sistema telefónico a una señal compatible la cual es enviada al microcontrolador para que éste cuente la cantidad de Ringing recibidos, una vez se llega a un valor preestablecido ( y en ausencia de un dispositivo como un contestador automático, fax o una persona), la tarjeta responde la llamada. El contestador de tonos se va decremenatando en $1, y$ una vez llega a cero, responde a la solicitud requerida.
Emulador de descuelgue

Este emulador contesta (como si levantáramos el auricular) y deja la llamada abierta estableciendo una conexión de tono entre los dos equipos.

\section{Identificador de clave o decodificador}

Este circuito decodifica una cadena de tonos DTMF (ver tabla 1) proveniente del teléfono, realiza la verificación de la clave, se encarga de "escuchar" permanentemente a la espera de un tono $y$, cuando lo recibe, lo decodifica, lo coloca en binario en las salidas Q0 a Q3 y acciona la salida Std. Esta última permanece activa tanto como dure el tono.

La tabla 1 de verdad explica en detalle el dato entregado correspondiente a cada uno de los tonos DTMF:

Para poder llevar a cabo la recepción de tonos DTMF se debe especificar con una lógica externa al MT $8880 \mathrm{cs}$ [14] el tiempo mínimo necesario para que un tono sea aceptado como valido. Es decir, el MT requiere de un arreglo externo para determinar el tiempo mínimo $\mathrm{T}_{\text {GTP }}$ que debe durar un tono para que sea decodificado. De manera similar, cuando termina la presencia de un tono valido, deberá transcurrir un tiempo $\mathrm{T}_{\mathrm{GTA}}$ para que el MT reconozca la ausencia del tono y esté listo para aceptar otro tono. 
Tabla 1. Sistema Multifrecuencial (DTMF)

\begin{tabular}{|c|c|c|c|c|}
\hline & & 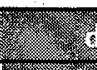 & & 28 \\
\hline Propar & olv & 312 & 92 & 80 \\
\hline 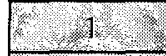 & 0 & 0 & 0 & 81 \\
\hline 2 & 0 & 0 & 1 & $\sqrt{0}$ \\
\hline 23 & 0 & 0 & 1 & $\sqrt{1}$ \\
\hline \begin{tabular}{|lll}
2 & 4 \\
\end{tabular} & 0 & 1 & 0 & .0 \\
\hline 5 & 0 & $\sqrt{1}$ & 0 & $\sqrt{1}$ \\
\hline 6. & 0 & 1 & 1 & 0 \\
\hline$\sqrt{7} 7$ & 0 & 21 & 1 & (1) \\
\hline$\sqrt{78}$ & 1 & 0 & 0 & 0 \\
\hline 9 & 1 & .0 & 0 & 41 \\
\hline 0 & 1 & 0 & 1 & 0 \\
\hline 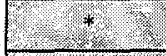 & 1 & 0 & 1 & 1 \\
\hline \# & 1 & 1 & 0 & 0 \\
\hline
\end{tabular}

\section{Sistema de encendido}

El sistema activa el switch de encendido del computador, entra a Internet y trata de comunicarse con la dirección IP del equipo origen, entonces se crea un socket y se inicia el intercambio de información entre los equipos.

La tarjeta va a permitir encender un equipo en forma remota, la cual deberá ser accedida a través de la línea telefónica y, debido a lo embebido de la tarjeta, el software incorporado tomará la decisión de qué rutina ejecutar cuando se produzca algún evento (ver cuadros 1 y 3 ).

\section{Funcionamiento de la tarjeta}

Es fundamental que el computador que se quiere gestionar esté conectado a la fuente de poder y a la conexión del canal telefónico para poder permitir el control de acceso y encendido. Dicha tarjeta es externa y esta conectada antes del MODEM de manera que tiene alimentación de la fuente de poder.

La tarjeta recibe datos en serie como la dirección IP y los 4 números de la clave, estos dígitos son deco- dificados (ver tabla 1) generando cierta cantidad de tonos que son detectados por la tarjeta para entender si es una llamada normal que se esta realizando o para encender el computador; si la llamada es para este fin, el equipo activa el subsistema emulador de descuelgue y verifica la clave. Si es correcta, es accionado el swicht de interrupción del encendido del computador y se ejecuta un software y captura la dirección IP que está en la tarjeta, entra a Internet y enrruta a dicha dirección estableciendo una comunicación (socket) [15] y envió de datos (escritorio remoto), ver cuadro 5 .

El servicio de la tarjeta es fallido si el computador no está conectado, si está encendido se obliga a entrar a Internet (ver cuadro 3); el/los equipo(s) a los que se conecte la tarjeta deben de tener las siguientes características mínimas: una torre (caja) XT, un MODEM, poseer sistema operativo Windows/linux (fedora2)

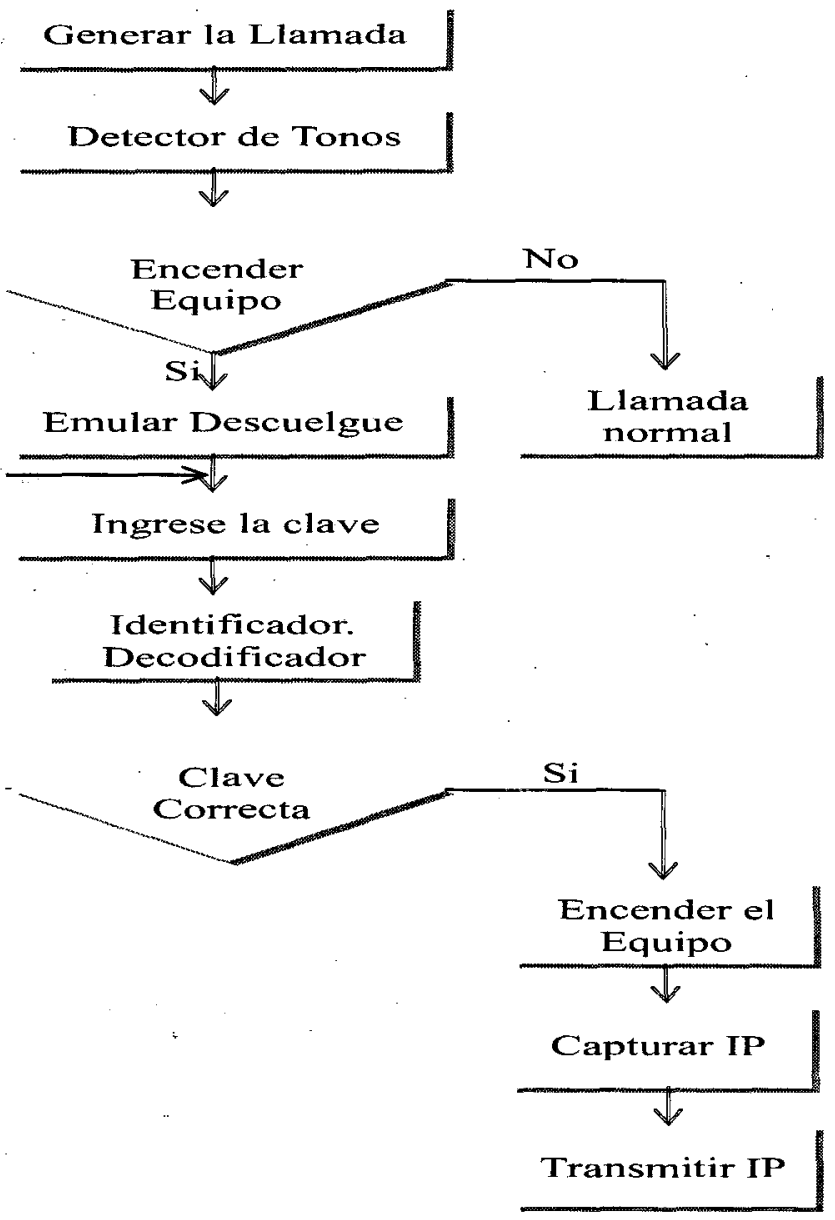

Figura 4. Diagrama de flujo. 
Cuadro 1. Eventos externos e internos.

\begin{tabular}{|c|c|c|c|c|}
\hline Evavio & Descricton & Mrewbors & 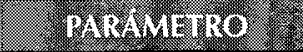 & pespusth \\
\hline Ringing & $\begin{array}{l}\text { Recepción de la } \\
\text { llamada }\end{array}$ & Usuario & $\begin{array}{l}\text { Numero de Ringing, } \\
\text { tonos programados. }\end{array}$ & $\begin{array}{l}\text { Tomar línea y escuchar } \\
\text { Tor }\end{array}$ \\
\hline Contrasena & $\begin{array}{l}\text { Solicitud de } \\
\text { autentificación. }\end{array}$ & Usuario. 8 & Clave de 4 dígitos. & Activación del sistema \\
\hline $\begin{array}{l}\text { Ingreso de la } \\
\text { contraseña al sistema }\end{array}$ & $\begin{array}{l}\text { Codificación de la } \\
\text { clave. }\end{array}$ & mitel : ? & $\begin{array}{l}\text { Pasar la señal } \\
\text { análoga a digital. }\end{array}$ & $\begin{array}{l}\text { Enviar tos dígitos al } \\
\text { microcontrolador }\end{array}$ \\
\hline $\begin{array}{l}\text { tiv? } \\
\text { Acinacion }\end{array}$ & $\begin{array}{l}\text { Activación del sistema } \\
\text { y acceso al sistema de } \\
\text { encendido }\end{array}$ & pic 16784 & $\begin{array}{l}\text { Autentificación de la } \\
\text { clave }\end{array}$ & $\begin{array}{l}\text { Alerta al sistema de } \\
\text { encendido }\end{array}$ \\
\hline Control: & Enciende el equipo & $\begin{array}{l}\text { Sistema de } \\
\text { encendido }\end{array}$ & Ninguno & Enciende \\
\hline
\end{tabular}

Cuadro 2. Respuestas del sistema frente a eventos externos.

\begin{tabular}{|c|c|c|}
\hline & brsantuctor & \\
\hline tomar línea & $\begin{array}{l}\text { El sistema toma la línea y escucha, } \\
\text { esperando el ingreso de la clave. }\end{array}$ & Numero de ring tones programados \\
\hline Activación del sistema & $\begin{array}{l}\text { El usuario ingresa la contraseña, } \\
\text { luego es decodificada. }\end{array}$ & Clave de 4 dígitos \\
\hline $\begin{array}{l}\text { Autentificación de la clave de } \\
\text { usuarifa? }\end{array}$ & $\begin{array}{l}\text { El microcontrolador recibe la clave y } \\
\text { obtiene la confirmación. }\end{array}$ & $\begin{array}{l}\text { Descodificación y autentificación de } \\
\text { la clave. }\end{array}$ \\
\hline Alerta al sistema de encendido & $\begin{array}{l}\text { Con la clave autentificada el pic da } \\
\text { la orden de encender el pc. }\end{array}$ & $\begin{array}{l}\text { Confirmaeión de comando de } \\
\text { control - encender }\end{array}$ \\
\hline Fin & Fin de la llamada. & Libera línea. \\
\hline
\end{tabular}

\section{Cuadro 3. Excepciones.}

\begin{tabular}{|c|c|c|}
\hline รरำ 10 & 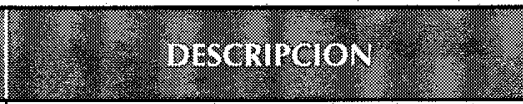 & 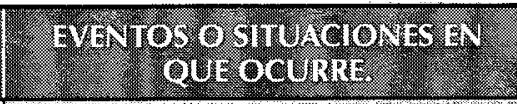 \\
\hline Contrasena no valida. & $\begin{array}{l}\text { La contraseña que ingresa es distinta } \\
\text { a la definida. }\end{array}$ & $\begin{array}{l}\text { Intento de ingreso no autorizado o } \\
\text { erróneo al sistema. }\end{array}$ \\
\hline Falla de linea & $\begin{array}{l}\text { La llamada se corta } \\
\text { inesperadamente }\end{array}$ & $\begin{array}{l}\text { El sistema se encuentra activo y el } \\
\text { sistema telefónico produce una falla } \\
\text { que corta la comunicación. }\end{array}$ \\
\hline
\end{tabular}


[16][17], memoria 64mhert, disco duro de 40m, procesador Pentium 3 en adelante.

Cuando se quiere comunicar o gestionar un computador [18] vía MODEM es indispensable considerar los siguientes factores: los computadores deben estar encendidos y tener el mismo sistema operativo (sistema de archivo) de lo contrario la comunicación es fallida.

El impreso de la tarjeta nos permite tener una visión más clara de la misma, permitiendo entender y conocer cada una de sus partes.

\section{Limitaciones de la tarjeta}

- La tarjeta no requiere de una línea telefónica adicional para su funcionamiento.

- Necesitara de una fuente de energía que la mantenga alerta para la encendida del PC.

- La tarjeta no permite controlar ni manipular un equipo de más de dos estados, solo lo encenderá y apagara.

- La tarjeta no alerta remotamente al usuario sobre eventos ni fallos ocurridos, ni tampoco es un contestador telefónico

- Se deberá desarrollar un software del lado del servidor o gestor, para el control de acceso e identificación de la tarjeta en el PC remoto donde la identificación debe estar regida bajo los estándares internacionales.

\section{Requisitos de interfaces}

Eventos externos a los que el sistema responde.

El sistema debe manejar una serie de excepciones:
El siguiente cuadro muestra las condiciones de excepción que se pueden dar en el sistema, en relación con eventos externos e internos.

La tarjeta estará regida por requisitos de calidad los cuales se especifican en forma cualitativa. El siguiente cuadro tiene las siguientes funciones:

Escala. Dimensión de medida.

Prueba. Método con que se hará en este caso específico la medición.

Peor. Valor apenas aceptable para el requisito (esto define el limite del fracaso).

Plan. Valor deseado para el requisito (esto define el limite del éxito).

\section{Conclusiones}

Con el diseño y elaboración de la tarjeta, el proyecto de investigación cumplió uno de sus objetivos fundamentales: desarrollar un dispositivo electrónico que a través de Internet encienda/apague un PC remoto para tener acceso a toda su información. Con su fabricación, se est estableciendo un uso masivo debido a su desempeño, eficiencia y productividad.

Se comprobó que dependiendo de la cantidad de tonos de marcado (4), la llamada tiene como fin encender el computador $y$, dependiendo de algún evento generado por el usuario (ingreso mal de la clave), genera una excepción. El funcionamiento de la tarjeta está basado exclusivamente para encendido/apagado del PC.

Con el desarrollo de la tarjeta se pretende que no todas las personas que conozcan el número telefónico de una persona determinada entren al equipo, ya que ésta cuenta con un identificador único. 
Cuadro 4. Requisitos de calidad de la tarjeta [19].

\begin{tabular}{|c|c|c|c|c|}
\hline $\begin{array}{l}\text { Caliofod de } \\
\text { Eumoiofallowat }\end{array}$ & rescala & 6. & -5 & (2) Plan \\
\hline Seguridad & $\begin{array}{l}\text { Cantidad de ingresos } \\
\text { indebidos }\end{array}$ & $\begin{array}{l}\text { Se realizarán como } \\
\text { máximo } 5 \text { intentos } \\
\text { con códigos de claves } \\
\text { inválidas }\end{array}$ & $\begin{array}{l}\text { Como máximo } 3 \\
\text { intentos }\end{array}$ & $\begin{array}{l}\text { Como máximo } 1 \\
\text { intento }\end{array}$ \\
\hline $\begin{array}{l}\text { Ejecución de } \\
\text { encendido }\end{array}$ & $\begin{array}{l}\text { Tasa de efectividad } \\
\text { de un buen } \\
\text { funcionamiento del } \\
100 \%\end{array}$ & $\begin{array}{l}\text { Se realizarán } 10 \\
\text { llamadas y se registrara } \\
\text { la respuesta del sistema } \\
\text { de cada una de ellas }\end{array}$ & Ninguno & Todos \\
\hline $\begin{array}{l}\text { Pérdida de línea } \\
\text { telefónica }\end{array}$ & $\begin{array}{l}\text { Depende } \\
\text { principalmente del } \\
\text { mediode transmisión y } \\
\text { está dado en segundos }\end{array}$ & $\begin{array}{l}\text { El usuario establece y, } \\
\text { corta la llamada cuando } \\
\text { crea necesario }\end{array}$ & $\begin{array}{l}\text { Depende de la } \\
\text { cantidad de veces } \\
\text { que el usuario lo } \\
\text { desée }\end{array}$ & Todos \\
\hline $\begin{array}{l}\text { Pérdida de energia } \\
\text { eléctrica }\end{array}$ & Está dado en segundos & $\begin{array}{l}\text { Contabilizarel } \\
\text { tiempo de activación } \\
\text { y desactivación de la } \\
\text { energia }\end{array}$ & $\begin{array}{l}\text { Depende de las veces } \\
\text { que se vaya la energía }\end{array}$ & $\begin{array}{l}\text { Depende de las } \\
\text { veces guie no se vaya } \\
\text { la energia }\end{array}$ \\
\hline Operativo & Será dada en segundos & $\begin{array}{l}\text { Contabilizar el tiempo } \\
\text { que se realiza el evento } \\
\text { hastra que se de la } \\
\text { respuesta al misno }\end{array}$ & Ninguno & $\begin{array}{l}\text { Todos } \\
\text { Tod }\end{array}$ \\
\hline
\end{tabular}

\section{Referencias bibliográficas}

[1] Stallings, W., (2000). Comunicaciones y redes de computadores, Sexta Edición, s.l.,Prentice Hall.

[2] Stallings, W. (2003). Redes e Internet de alta velocidad - rendimiento y calidad de servicio, $2^{\mathrm{a}}$ edición, s.l., Ed. Prentice hall.

[3] Carballar J. A. (1996), El Libro de las comunicaciones del PC, España, Ed. Alfaomega Ra-ma.

[4] www.setsi.mityc.es/normali/normaliz/uit. htm

[5] Jame S. Kurose, Keith W. R. (2001). Computer Networking, s.l., Pearson Education. Libro sobre Internet y sus protocolos.

[6] Huidobro J., Roldan D. (2003), Integración de voz y datos, España, Ed. Mc Graw Hill.

[7] Comer, D. E. (1996). Redes globales de información con Internet $y \mathrm{TCP} / \mathrm{IP}$, vol. 1 principios básicos, protocolos y arquitectura, $3^{\text {a }}$ edición, s.l., Ed. Prentice Hall.

[8] García T. J. (2002). Alta velocidad y calidad de servicio en redes IP, España, Rd Ra-ma.

[9] Hakala D. (1998), MODEM a su alcance, s.l., Ed Mc Graw Hill.

[10] Barry B. Brey. (2001). Los microprocesadores Intel, 5a ed., México D.F., Ed Prentice Hall.

[11] Murray William H., (1987), Programación en Lenguaje ensamblador 80386/80286", s.l., Ed. McGraw Hill.

[12] www.htmlweb.net/redes/tcp_ip/capa_2/ acceso_red_1.html

[13] León García, A.; Widjaja, I., (2002) Redes de comunicaciones. Conceptos fundamentales y arquitecturas básicas, McGraw Hill. 
[14] Mitel. (1997). Iso'-Cmos MT8880C integrated DTMF transceiver.

[15] Commer, D. E. (2000). Interconectividad de redes con tcp/ip, vol 2: Diseño e implementación. Ed. Pentice Hall.

[16] Peterson Richard, (2001), Manual de referencia Linux, s.l., Ed McGraw Hill.
[17] Eyler, P. (2001). Redes Linux con tcp/ip., s.l., Ed. Prentice Hall.

[18] Huidobro J., Roldan D. (2004), Redes y servicios de banda ancha, España, Ed. McGraw Hill.

[19] Gilb T. (2005), Competitive Engineering, disponible en www.tom gilb.com 